\title{
Energy efficiency of coordinated multipoint transmission (CoMP) over LTE-A
}

\begin{abstract}
Coordinated Multipoint (CoMP) is one of the new technologies introduced in Long Term Evolution-Advanced (LTE-A) which helps to achieve the requirements of $4 \mathrm{G}$ issued by International Telecommunication Unit (ITU). At the same time, it is also one of the techniques for Inter-cell Interference Coordination (ICIC) that assists to eliminate the interference caused by neighboring base stations. Meanwhile, it also able to improve the channel capacity of the system. In brief, CoMP can be implemented for user experiences weak signals especially user at the cell edge because CoMP will coordinate several base stations for transmission. However, there is some challenges that CoMP technique faced which is energy efficiency and this is the main focus of this project because it always becomes ignorance. This project is mainly analyze the energy efficiency of the system by comparing the system with CoMP and non-CoMP or conventional system. There are few types of power consumption's considered which are transmit power, base station power and backhaul power. Therefore, a generic analytical equation is used for simulation conducted in this project. The energy efficiency is in the unit of bits/joule. Via the simulation, a system with best energy efficiency is identified. Besides that, analysis of the results is discussed in details. Other than that, there is further analysis on the system with best energy efficiency. The portion of each type of power consumed from the total power consumption is calculated. Furthermore, there is some suggestions on reducing the power that consumed the most because this kind of power has high potential on further improves the energy efficiency of the system. In addition, this helps to move the world towards green technology path smoother or less barrier.
\end{abstract}

Keyword: CoMP; LTE-advanced; Energy efficiency 